Apsilidisl, N.; ASCE, S. M.; Diplas, Panayiotis; ASCE, M.; Dancey, C. L.; Vlachos, P. P.; Raben, S. 0.

Local Scour At Bridge Piers: The Role Of Reynolds Number On Horseshoe Vortex Dynamics

Verfügbar unter / Available at:

https://hdl.handle.net/20.500.11970/100205

Vorgeschlagene Zitierweise / Suggested citation:

Apsilidisl, N.; ASCE, S. M.; Diplas, Panayiotis; ASCE, M.; Dancey, C. L.; Vlachos, P. P.; Raben, S. O. (2010): Local Scour At Bridge Piers: The Role Of Reynolds Number On Horseshoe Vortex Dynamics. In: Burns, Susan E.; Bhatia, Shobha K.; Avila, Catherine M. C.; Hunt, Beatrice E. (Hg.): Proceedings 5th International Conference on Scour and Erosion (ICSE-5), November 7-10, 2010, San Francisco, USA. Reston, Va.: American Society of Civil Engineers. S. 86-94. 


\title{
Local Scour At Bridge Piers: The Role Of Reynolds Number On Horseshoe Vortex Dynamics
}

\author{
N. Apsilidis ${ }^{1}$, S.M. ASCE, P. Diplas ${ }^{2}$, M.ASCE, C.L. Dancey ${ }^{3}$, P.P. Vlachos ${ }^{4}$ and \\ S.G. Raben ${ }^{5}$
}

Baker Environmental Hydraulics Laboratory, Department of Civil and Environmental Engineering, Virginia Polytechnic Institute and State University, Blacksburg, VA 24061-0105, PH (540) 231-2357, e-mail: napsilid@avt.edu

2 Baker Environmental Hydraulics Laboratory, Department of Civil and Environmental Engineering, Virginia Polytechnic Institute and State University, Blacksburg, VA 24061-0105, PH (540) 231-6069, e-mail: pdiplas@vt.edu

3 Baker Environmental Hydraulics Laboratory, Department of Mechanical Engineering, Virginia Polytechnic Institute and State University, Blacksburg, VA 24061-0238, PH (540) 231-7466, e-mail: cldra vt.edu

4 Advanced Experimental Thermofluid Engineering Research Laboratory, Department of Mechanical Engineering, Virginia Polytechnic Institute and State University, Blacksburg, VA 24061, PH (540) 231-3366, e-mail: pvlachos@v,t.edu

5 Advanced Experimental Thermofluid Engineering Research Laboratory, Department of Mechanical Engineering, Virginia Polytechnic Institute and State University, Blacksburg, VA 24061, PH (540) 231-7187, e-mail: sraben@vt.edu

\begin{abstract}
This paper reports a comprehensive study of the major scour agent around bridge piers: the turbulent horseshoe vortex. The intricate and inherently unsteady characteristics of the junction flow are captured within a series of scaled laboratory experiments. We applied the state-of-the art Time-Resolved Digital Particle Image Velocimetry (TR-DPIV) technique to measure the velocity field at the centerline plane of symmetry upstream of a cylindrical model. Three levels of Reynolds numbers $\left(\mathrm{Re}_{\mathrm{D}}\right)$ based on the obstacle diameter were studied: 26,000, 48,000 and 117,500 . We evaluated the effect of this factor based on the time-averaged analyses of velocity and vorticity. Basic statistical analysis of the fluctuating velocity components provided insight to the physical mechanism that governs the behavior of the horseshoe vortex at the aforementioned levels of $\mathrm{Re}_{\mathrm{D}}$.
\end{abstract}

\section{INTRODUCTION}

Erosion of the loose boundary material around bridge piers poses a significant threat to the structural integrity of the bridge. Past studies have identified hydraulic scour as the major cause of bridge failures (Wardhana and Hadipriono, 2003 \& Briaud and Hunt, 2006). As a result, a large number of scour-related studies have been documented in the literature (for a comprehensive list, refer to Melville and 
Coleman, 2000). Despite the intense research activity, we are still lacking satisfactory answers regarding the dominant characteristics of the scour mechanism.

We put forward that the key in this effort is the study of the intricate system of vortices, which forms at the juncture base and stretches around the obstacle. Named after its shape, the horseshoe vortex is the result of the interaction between the approaching boundary layer and the adverse pressure gradient imposed by the body (Simpson, 2001). In this case, the disturbed flow experiences a three-dimensional separation and the entrained vorticity rolls up to form the vortical system under investigation.

In a pioneering experimental study, Baker (1979) was among the first to evaluate the dynamics of a turbulent boundary layer propagating along a flat plate. His work revealed the topological characteristics of the flow at the upstream centerline plane of symmetry using oil visualizations. In this way, he documented the instantaneous presence of a four-vortex system. Moreover, these experimental data validated the results of dimensional analysis, according to which the position of the main vortex is expressed as a function of the boundary layer displacement thickness $\delta^{*}$ and the Reynolds number based on the body diameter, $\operatorname{Re}_{\mathrm{D}}$. The range of the second parameter was between 4000 and 90,000.

A decade later, another experimental work by Dargahi (1989) enhanced the state of knowledge with velocity measurements collected using a hot-film anemometer. For $20,000<\mathrm{Re}_{\mathrm{D}}<39,000$, a system of at least five vortices was identified. Hydrogen bubble visualizations highlighted the trend for increased flow complexity with $\mathrm{Re}_{\mathrm{D}}$. The existence of a positive relation between the size of vortex system and the Reynolds number was also reported.

Devenport and Simpson (1990) provided a further insight to the physics of the turbulent horseshoe vortex. They applied the high temporal resolution Laser Doppler Anemometry technique using air as the working fluid. Their analysis disclosed the intermittent character of the turbulent flow for $\mathrm{Re}_{\mathrm{D}}=115,000$. Two modes were identified: the backflow and the zero-flow. During the first one, the deflected fluid forms a jet that penetrates the vortex region and propagates toward the upstream. On the other hand, when the zero-flow mode is active, the aforementioned motion is inhibited and finally the jet is ejected upwards. These states were documented in the probability density functions of the streamwise and vertical velocity components, where two distinct peaks were identified. Flow switches between the two modes in a seemingly random manner and this transition is believed to play an important role in the generation of increased turbulent stresses.

Similar findings regarding the quasi-periodic nature of the vortex flow were reported by Agui and Andreopoulos (1992). In this study emphasis was placed on obtaining time-resolved measurements of the wall pressure for two levels of $\mathrm{Re}_{\mathrm{D}}$ at 100,000 and 220,000 . Their major finding is the existence of bimodal shapes for the root mean square pressure fluctuations in two regions of the flow not previously identified: close to the separation line and in the wake of the cylinder. 
Despite the remarkable progress that has been made in the study of the horseshoe vortex dynamics and the unsteady characteristics of junction flows, a comprehensive study that would incorporate the latest advances in experimental fluid mechanics is still lacking. The present work seeks to augment the current state of knowledge about this phenomenon. In order to accomplish our goal, we conducted a series of high spatio-temporal resolution experiments employing the global flow measurement TR-DPIV technique. The investigation was performed for three levels of $\mathrm{Re}_{\mathrm{D}}$ corresponding to turbulent junction flows: $26,000,48000$ and 117,500. All measurements reported herein refer to the upstream centerline plane of symmetry.

\section{PHYSICAL MODEL}

Experiments were carried out in a recirculating water tunnel. The total length of the channel is $8.74 \mathrm{~m}$, however the test section has dimensions of $1.81 \mathrm{mx} 0.61 \mathrm{~m}$ x $0.61 \mathrm{~m}(\mathrm{~L} \mathrm{x} \mathrm{W} \mathrm{x} \mathrm{H})$. The bottom and side walls of this section are made of clear acrylic plexiglass to facilitate optical access for the implementation of DPIV technique. Water was initially pumped into a settling chamber, where a set of screens and a 6:1 three-dimensional contraction were used to deliver swirl-free flow.

At the entrance of the test section, we installed a $10 \mathrm{~cm}$ wide sand strip so as to trip the flow and bring about the development of a turbulent boundary layer at the location of the cylinder. The data sets were recorded at the junction between the flat bottom and the protuberance at an average distance of $1.55 \mathrm{~m}$ from the same reference point.

Three cylindrical PVC pipes of diameters $0.06 \mathrm{~m}, 0.089 \mathrm{~m}$ and $0.168 \mathrm{~m}$ were used to generate the horseshoe vortex system for the corresponding levels of Reynolds numbers. Each cylindrical body was mounted vertically on the bottom of the channel (Figure 1).

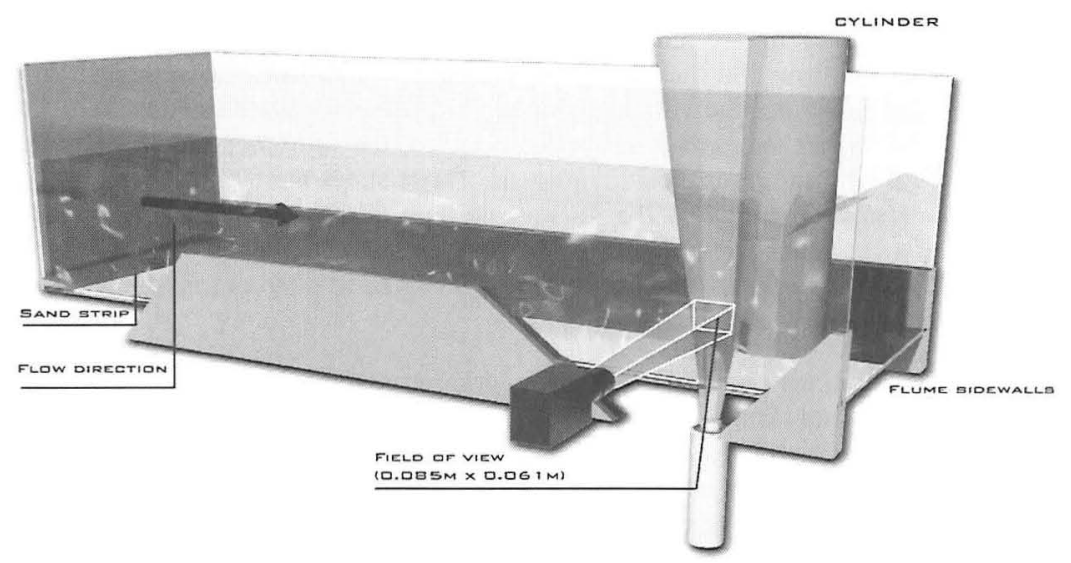

Figure 1. View of the experimental setup 
The main factors together with their levels for each experiment are summarized within Table 1.

Table 1. Experimental matrix

\begin{tabular}{|ccccccc|}
\hline Experiment & $\mathbf{U}(\mathbf{m} / \mathbf{s})$ & $\mathbf{H}(\mathbf{m})^{*}$ & $\mathbf{D}(\mathbf{m})$ & $\mathbf{F r}^{*}$ & $\mathbf{R e}_{\mathbf{H}}$ & $\mathbf{R e}_{\mathbf{D}}$ \\
\hline $\mathbf{1}$ & 0.40 & 0.15 & 0.060 & 0.33 & 64,500 & 26,000 \\
\hline $\mathbf{2}$ & 0.50 & 0.18 & 0.089 & 0.38 & 96,770 & 48,000 \\
\hline $\mathbf{3}$ & 0.65 & 0.25 & 0.168 & 0.42 & 174,130 & 117,500 \\
\hline
\end{tabular}

* $\mathrm{H}$ is the flow depth and Fr the Froude number

\section{INSTRUMENTATION}

The DPIV system consisted of a double-pulsed Nd: YAG laser source of green wavelength at $532 \mathrm{~nm}$. The laser beam was shaped into a planar sheet of approximately $2 \mathrm{~mm}$ thickness. The light was delivered from the bottom of the flume. Images were taken with an IDT X-3 CMOS high-speed camera. Prior to each data collection session, the optical axis of the camera was carefully aligned until it became perpendicular to the laser plane. To control the operation of the aforementioned pieces of equipment, we connected them to a synchronizer that was monitored through a PC software interface.

Water was seeded with silver-coated hollow glass bead particles with a specific gravity very close to 1 and a mean diameter of $135 \mathrm{um}$. Given these numbers, the tracers are exhibiting a neutrally buoyant flow behavior. Seeding particles were incrementally introduced into the flow, until we achieved a density of 10-12 particles within an interrogation area of $32 \times 32$ pixels.

The magnification factor between the real and the image plane was 63.5 um/pixel. To estimate this value, we took pictures of a steel ruler placed at the centerline of the laser sheet. The physical dimensions of the field of view were $8.5 \mathrm{~cm}$ x $6.1 \mathrm{~cm}$. A total of 3270 images with $1280 \times 1024$ pixels resolution were collected at a sampling rate of $1 \mathrm{kHz}$. The time interval between pulses was $1 \mathrm{~ms}$.

Pre-processing of the PIV data consisted in masking areas of the image that did not include velocity information (solid boundaries). Consequently, we evaluated the raw data by applying a multi-grid, discrete window offset analysis (Westerweel and Dabiri, 1997) in two passes. During the first one, the dimensions of the interrogation window were $64 \times 64$ pixels resulting in a grid resolution of $16 \times 16$ pixels. In the second pass, the interrogation window was reduced to $32 \times 32$ pixels, in order to capture the minute details of the flow. Subsequently, the final grid resolution was increased to $8 \times 8$ pixels. In both cases, a Gaussian window was used (Eckstein and Vlachos, 2009) the statistical correlation of image intensities was performed with the Robust Phase Correlation technique (Eckstein et al., 2008). Finally, we validated the flow field applying the Universal Outlier Detector technique of Westerweel and Scarano (2005). In this way, the spurious velocity vectors were identified and substituted with neighboring averages. The final spatial resolution of the velocity field was $0.51 \mathrm{~mm}$ and the total number of velocity vectors 20,193 . 


\section{RESULTS AND DISCUSSION}

\section{Time-averaged data analysis}

The application of DPIV technique in experimental fluid mechanics yields a vast amount of collected data. Time-averaged analysis provides a concise and efficient way to represent them. For this reason, Figure 2 illustrates contour plots of the streamwise velocity component (expressed in $\mathrm{m} / \mathrm{s}$ ) for each $\mathrm{ReD}$ case. The average path of seeding (and consequently fluid) particles is documented by the steamlines of the flow field. To facilitate comparison, the streamwise and vertical dimensions have been normalized with the characteristic length (cylinder diameter).
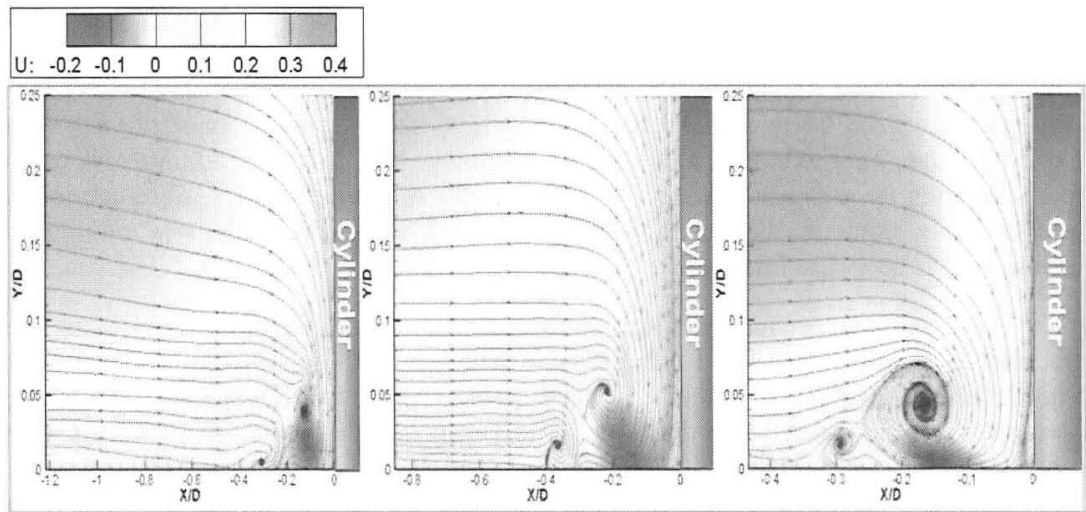

Figure 2. Time-averaged streamwise velocity component $U$ for $\operatorname{Re}_{D}$ : 26,000, 48,000 and 117,500

Streamline curvature is indicative of a dominant vortical structure for all three cases. This primary vortex (PV) is characterized by the motion of fluid elements in a clockwise sense. Its average position varies in the $\mathrm{x}$-direction for each Reynolds number, but the values do not indicate a specific trend $\left(X / D=-0.12\right.$ for the small $\operatorname{Re}_{\mathrm{D}}$, -0.21 for the middle and -0.16 for the large) .

Moreover, in all cases a secondary vortex (SV) is identified further upstream of the PV. Evidence of a smaller vortex at the corner is provided only for the case of the $\operatorname{Re}_{\mathrm{D}}=117,500$. These results are partially in agreement with the topological analysis of junction flows reported by Hunt et al. (1978). In their study, they propose that a tertiary vortex (TV) forms between the PV and the SV and is rotating in a counter-clockwise sense. Our data do not support the existence of such a vortex with steady space-time characteristics.

Time-averaged, spanwise vorticity contours are displayed in Figure 3 (units are in $\left.\mathrm{s}^{-1}\right)$. Areas of high negative vorticity coincide with high circulation regions that constitute the cores of the PV. Apart from the core vorticity, two distinct vorticity 
patterns are identified. The first is expressed as a tail of negative vorticity that follows the core. This feature is more prominent for the lowest $\mathrm{Re}_{\mathrm{D}}$, where higher vorticity levels were calculated. The second vorticity pattern is located directly below the PV core. Its existence showcases the interaction between the horseshoe vortex and the solid boundary. Paik et al. (2007) elaborated on the importance of this structure for the eruption of wall vorticity and its contribution to the unsteady nature of the vortex.

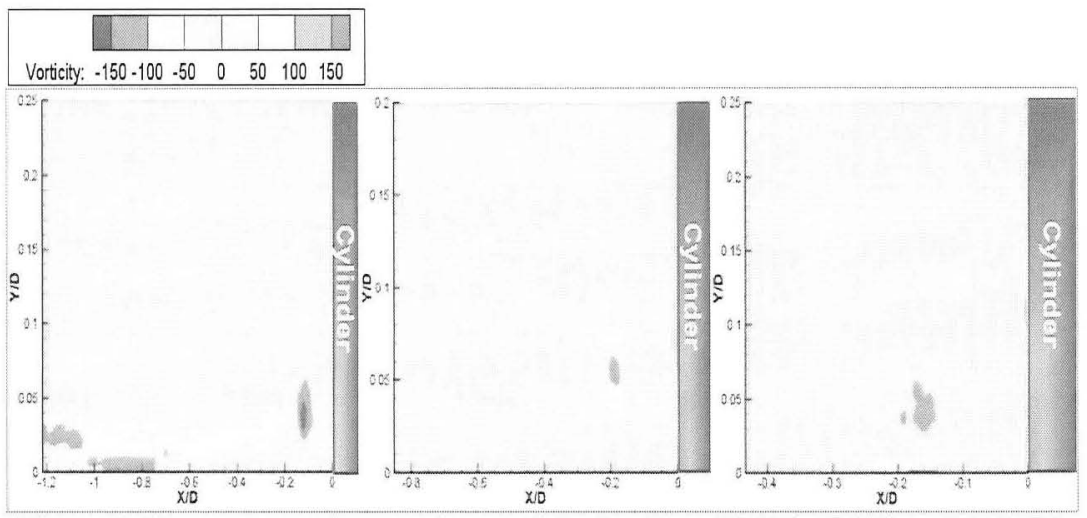

Figure 3. Time-averaged spanwise vorticity for $\operatorname{Re}_{\mathrm{D}}: 26,000,48,000$ and 117,500

\section{Statistical analysis}

Probability density functions (pdf) of both fluctuating velocity components were derived along vertical lines that cross the core of each vortex. Due to the high spatial resolution of DPIV measurements, we were able to reproduce a large number of such plots. However, due to limitations in space, we include here specific cases of the streamwise $(\mathrm{u})$ fluctuations, which are representative of the overall trends. Normalization is performed with the root mean square velocity urms.

The oscillating behavior of horseshoe vortex system between the two modes described in the introductory section is documented with the existence of two peaks. The backflow mode manifests itself by a peak in the area of negative velocities, whereas the zero-flow mode is accompanied with a peak in the positive axis. These characteristics are observed at the region demarcated from the lower streamlines that constitute the PV and the solid boundary. Outside this area, the pdf curves are unimodal.

The aforementioned trend was not observed for the case of the lower $\mathrm{Re}_{\mathrm{D}}$. Figure 4 consists of plots that are characterized by a one-peak distribution. Data are approximately centered around zero. On the other hand, bimodal shapes are present in Figure 5 (corresponding to $\mathrm{Re}_{\mathrm{D}}=48,000$ ). In this case, the negative peak is greater than the positive one, underscoring the dominance of the backflow state in this 
continuous interplay of modes. However, the strongest evidence for bimodality is shown in Figure 5. For the highest Reynolds number, the size of the positive peak is comparable to that of the negative one. Therefore, the contribution of the zero-flow mode in the dynamics of high-Reynolds number flows cannot be neglected.
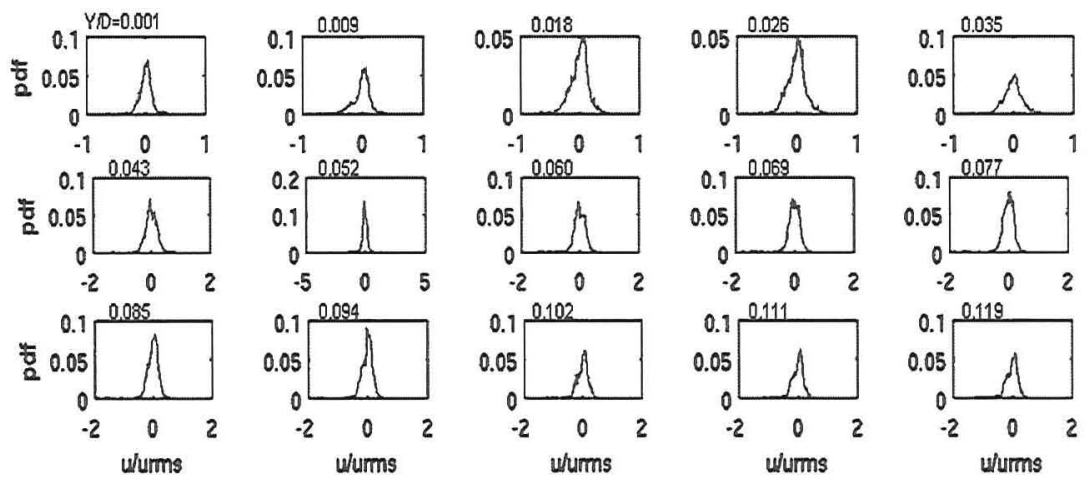

Figure 4. Probability density functions for the u fluctuating component at streamwise location $X / D=-0.12\left(\operatorname{Re}_{D}=26,000\right)$
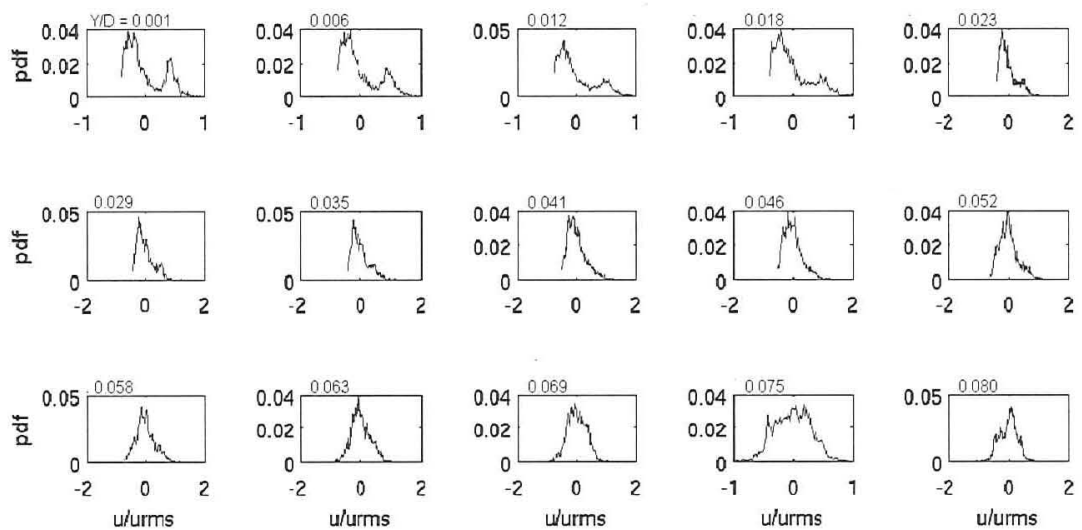

Figure 5. Probability density functions for the u fluctuating component at streamwise location $X / D=-0.21\left(\operatorname{Re}_{D}=48,000\right)$ 

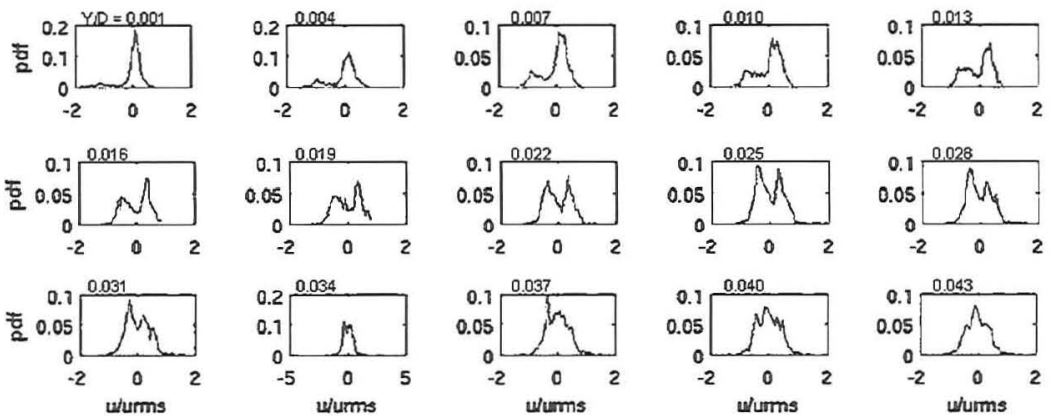

Figure 6. Probability density functions for the u fluctuating component at streamwise location $X / D=-0.18\left(\operatorname{Re}_{D}=117,500\right)$

\section{CONCLUSIONS}

The main purpose of this experimental study was to test the hypothesis that the Reynolds number plays a role in shaping the dynamics of the turbulent horseshoe vortex. Through time-averaged analysis we were able to gain insight into the prevailing characteristics of important flow parameters such as velocity, vorticity and turbulent kinetic energy. As far as the topological characteristics of the flow are concerned, we discovered similarities regarding the structure of the vortex systems. Nevertheless, the distributions of velocity constituents at the region below the horseshoe vortex are revealing that the bi-modal instability is not a universal characteristic of junction flows.

In conclusion, this work adds to the state of knowledge of junction flows in the vicinity of a cylindrical model pier by revealing the dependency of the horseshoe vortex dynamics on $\mathrm{Re}_{\mathrm{D}}$. This finding will guide future experimental work seeking to investigate bridge pier flow characteristics in the presence of a fully-rough, erodible boundary. Sediment transport data will be integrated with time-resolved measurements collected for the present study to validate a numerical model capable of accurately reproducing bridge foundation scour. Once developed, this advanced tool will serve as a valuable resource to engineers toward the safe and financially sound design of bridge foundations.

\section{ACKNOWLEDGEMENTS}

Our work was supported by the National Science Foundation (EAR 0738759) and the Research Office of the U.S. Army Corps of Engineers (ARO 53512-EV). The first author would also like to thank the Academy of Athens (Greece) for providing him financial support through the bequest of Praksitelis and Sofia Argyropoulos. 


\section{REFERENCES}

Agui, J. H., and Andreopoulos, J. (1992). "Experimental investigation of a threedimensional boundary layer flow in the vicinity of an upright wall mounted cylinder." Journal of Fluids Engineering 114: 566-576.

Baker, C. J. (1979). "The turbulent horseshoe vortex." Journal of Wind Engineering and Industrial Aerodynamics 6: 9-23.

Briaud, J.L. and Hunt, B.E. (2006). "Bridge scour \& the structural engineer." Structure magazine 13 (12): 58-61.

Dargahi, B. (1989). "The turbulent-flow field around a circular cylinder." Experiments in Fluids 8: 1-12.

Devenport, W. J., and Simpson, R. L. (1990). "Time-dependent and time-averaged turbulence structure near the nose of a wing body junction." Journal of Fluid Mechanics 210: 23-55.

Eckstein, A., Charonko, J., and Vlachos, P.P. (2008). "Phase correlation processing for DPIV measurements." Experiments in Fluids 45: 485-500.

Eckstein, A., and Vlachos, P. P. (2009). "Assessment of advanced windowing techniques for digital particle image velocimetry (DPIV)." Measurement Science \& Technology, 20(7): 075402.

Hunt, J.C.R., Abell, C.J., Peterka, J.A., and Woo, H. (1978). "Kinematical studies of the flows around free or surface-mounted obstacles; applying topology to flow visualization." Journal of Fluid Mechanics 86: 179-200.

Melville, B.W. and Coleman, S.E. (2000). Bridge Scour, Water Resources Publications, Littleton, Colo.

Paik, J., Escauriaza C., and Sotiropoulos, F. (2007). "On the bimodal dynamics of the turbulent horseshoe vortex system in a wing-body junction." Physics of Fluids 19(4): 045107.

Simpson, R. L. (2001). "Junction flows." Annual Review of Fluid Mechanics (33): 415-443.

Wardhana, K., and Hadipriono, F. C. (2003). "Analysis of recent bridge failures in the United States." Journal of Performance of Constructed Facilities, 17(3): 144-150.

Westerweel, J., Dabiri D., and Gharib M. (1997). "The effect of a discrete window offset on the accuracy of cross-correlation analysis of digital PIV recordings." Experiments in Fluids 23: 20-28.

Westerweel, J., and Scarano F. (2005). "Universal outlier detection for PIV data." Experiments in Fluids 39: 1096-1100. 